\title{
How to identify Airport Conflict Hot Spots
}

\author{
Zheng-hong Xia, Wei-jun Pan \\ (School of Air Traffic Control, Civil Aviation Flight University of China, Guanghan 618307)
}

\begin{abstract}
Keywords: Hot Spots; Conflicts Identification; Airport Surveillance Radar (ASR)
Abstract: For those aerodrome areas where collision between aircraft may happen, based on the Airport Surveillance Radar (ASR) data, the taxi tracks of aircraft in the aerodrome maneuver area were extracted. The method of judging whether the taxi route and taxi time of any two aircrafts which go through the same taxiway is overlapped, whether the absolute value of the difference of headings and the separation satisfy the constraint condition of the hot-spot identification in airport was proposed. According to result of conflict Hot Spots identification, it is consistent with the experts' feedback opinions and the practical operation situation, which is verified the rationality of airport conflict Hot Spots identification method.
\end{abstract}

\section{Introduction}

Recently, rapidly growing demand for air transportation has resulted in increasingly complicated runways and taxiways with more intersections. The amount of accidents and potential incident proneness due to taxiing error between two aircrafts or an aircraft and a vehicle increase daily. Owing to unreasonable airport layout, confusing signs, inappropriate light setting or human factors, areas or intersection that tends to confuse the aircrew or induce taxiing errors is defined as airport conflict Hot Spots.

The term Airport Conflict Hot Spots is defined as "maneuver areas in airport where there used to be, or may be the risks of runway collision or intrusion, and where pilots and ground vehicle drivers should enhance vigilance" in Procedures for Air Navigation Service-Air Traffic Control (Doc 4444) by ICAO (International Civil Aviation Organization). Based on the historical accidents and incident proneness, the method of how to identify the conflict areas was proposed. And FAA (Federal Aviation Administration, USA) furthers pointed out that the airport conflicting Hot Spots tend to be the intersection of two taxiways, or a runway and a taxiway. Meanwhile, circles and polygons are recommended to mark all Hot Spots in the airport chart. Research on airport conflict Hot Spots identification is still under incipient stage in China. Huo Zhi-qin screen and identify the conflict areas based on the history unsafe occurrences using Triple Selection Method, whose difficulty is how to obtain sufficient sample cases of an airport. Therefore, the method of inquiring pilots, controls and car drivers to indentify conflict areas is commonly used at present. However, as the existential discrepancy of layers and positions, the accuracy and rationality of the test results will be questioned.

Based on the Airport Surveillance Radar data, the history taxi tracks of aircrafts were extracted, and then the unsafe events features in space and time was analyzed on the basis of speed, heading and position variations. The principles that whether aircrafts which taxi through the same taxiway has the coincidence taxi route and coasting time, whether the angle difference and distance separation dissatisfy with threshold value were proposed in this paper. According to the case study, the position of conflict areas which was identified with this approach was the same as the experts' opinion and the practical control operation.

\section{Preprocess Airport Surveillance Radar Data}

The approaches of monitoring the airport operation include camera, ADS-B (Automatic Dependence Surveillance - Broadcasting), ASR (Airport Surveillance Radar) and Multipoint Orientation. And the 
ASR has relatively higher precession and faster data refresh rate. It records the taxiway, time, position and heading of every ground movement targets, which provides great guarantee of surveillance of the aircrafts and vehicles movements. Therefore, the ASR data can be used for detecting and recognition of conflict Hot Spots. First, the ground taxi tracks of aircrafts were withdraw from the ASR data, and then the conflict areas of the airport was identified with the principles listed in the following section, which will be testified by experts' feedback opinions. Finally, the position of conflict Hot Spots will be marked on the airport chart.

The tracks of movement targets on the airport were recorded in the Airport Surveillance Radar. However, only the aircrafts taxi tracks were analyzed for identifying the history or potential conflicts in the maneuver areas of airport. Therefore, the tracks of vehicles and tracks of aircrafts in the air were excluded by data preprocess. One row of the record will be read, if the record is one aircraft track, and it moved on the maneuver ground area. And then query the aircraft tracks object set

TRACK $=\left\{\right.$ track $_{1}$, track $_{2} \ldots$, track $\left._{n}\right\}$ for the existence of one aircraft by its call-sign and time. If not exits, create a new object track, and then this record will be added to track, which is inserted into TRACK . where the object track contains the track points set $T R$ and the passed taxiway set $T L$.Each track point $t r$ includes the longitude $\operatorname{pos}_{x}^{t r}$ and latitude $\operatorname{pos}_{y}^{t r}$, speed and heading, the first time of track existence $T^{\text {satr }}$ and the time span $T_{\text {taxi }}$ from the beginning to concrete track. And $T L$ includes the taxiways where one aircraft has passed. Each taxiway records the first entrance track point $t r^{\text {ener }}$ and last exit track point $t r^{\text {exit }}$. Finish this preprocess until the record file was read to end. Finally, the taxiway layer of digital map was traversed for the taxiway $\operatorname{set} T W=\left\{t w_{1}, t w_{2} \ldots, t w_{m}\right\}$.

\section{Algorithm for Identifying Conflict Hot Spots}

The key ideal of the algorithm for identifying conflict Hot Spots can be seen from figure. Obtain one taxiway named $t w_{i}$, and transverse the aircraft tracks set $T R A C K$, then insert the aircraft object into set $T P_{i}$ according to its passed taxiway set $T L$.Arbitrarily Get two aircrafts named $p_{j}$ and $p_{k}(j<k)$, calculate whether the exit time of $p_{j}$ is coincidence with the enter time of $p_{k}$ in the taxiway of $t w_{i}$. If so, analyze whether the heading difference $H D_{j, k}$ is larger than the threshold value named $H D$ offset . And suppose the time span from $p_{k}$ enter into $t w_{i}$ without holding to the exit time of $p_{j}$, calculate the distance named $D i s_{j, k}$ between the track points of $p_{j}$ and $p_{k}$ is smaller than the threshold value named Disoffset. If so, the conflict area between $p_{j}$ and $p_{k}$ was detected for Hot Spot named $h s_{t w_{i}}$. If not, the value $\mathrm{j}$ and $\mathrm{k}$ will be added with 1 . And if the conflict area $h s_{t w_{i}}$ has existed in the Hot Spots set $H S$, the two aircrafts $p_{j}$ and $p_{k}$ should be insert into the conflict plane list $H P$. Or else create on Hot Spot object $h s_{t w_{i}}$ with the two aircrafts in its conflict plane list $H P$.

$$
\begin{gathered}
H D_{j, k}=\left|h d_{j}-h d_{k}\right| \\
D i s_{j, k}=\sqrt{\left(p o s_{x}^{r^{\prime}}-p o s_{x}^{t^{k}}\right)^{2}+\left(p o s_{y}^{t r^{\prime}}-p o s_{y}^{t^{k}}\right)^{2}}
\end{gathered}
$$




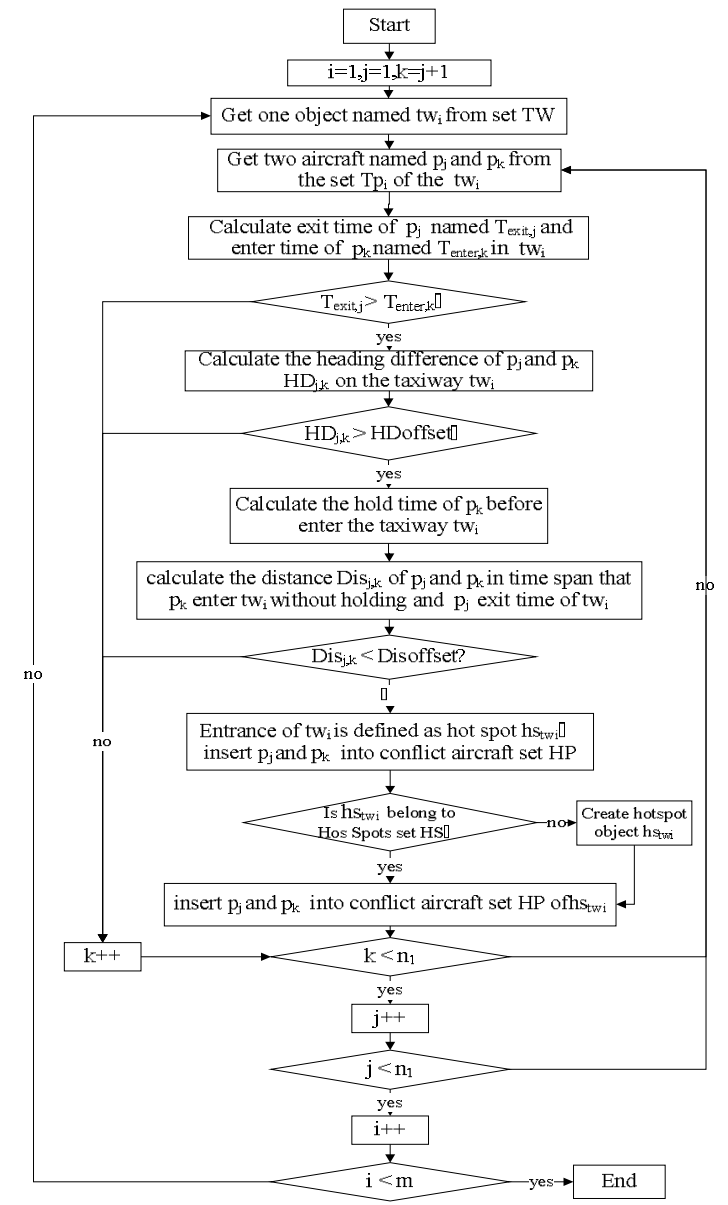

Figure.1 The flow chart of algorithm for identifying airport conflict Hot Spots

\section{Case Study}

The ASR data used for identifying Hot Spots in this paper was one week data from December $9^{\text {th }}$ to $15^{\text {th }}, 2012$, which was originated from one Chinese airport whose traffic flow was the top three of China. The traffic flow of this week is summed up to 5820 sorties and take-off and landing 830 sorties daily on average, it contains about 12010685 discrete track points. Two runways with magnetic heading of $016^{\circ}$ and $196^{\circ}$ are located in this airport. The west one runway's length is 3800 meters while the east one 4000 meters, and the runways separation is 2200 meters. Therefore, aircrafts can takeoff or landing on the runway independently. Based on the aforementioned conflict detecting algorithm, the threshold value HDoffset $=90^{\circ}$, Disoffset $=100 \mathrm{~m}$. The airport conflict Hot Spots identification results can be obtained, then the experts and operational officers were organized to testify its rationality. Finally, the locations of conflict Hot Spots were marked on the airport chart, which can remind the pilots and car drivers, pay special attention to these dangerous areas, reduce or completely eradicate the ratio of runway incursion and ground collisions, and enhance the safety operational level of aerodrome effectively. 


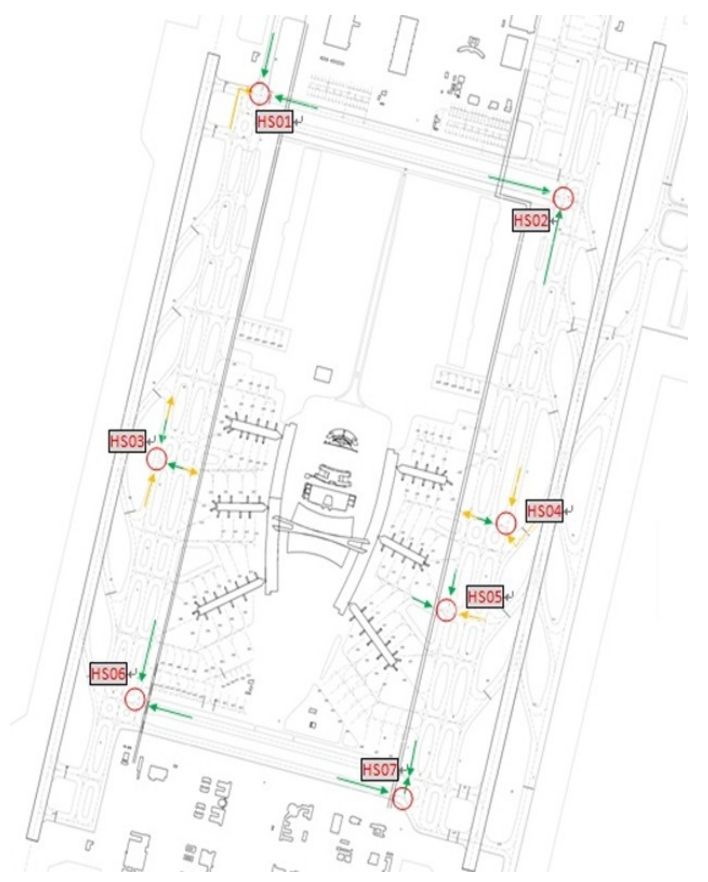

Figure.2 Airport conflict Hot Spots identification results

\section{Conclusions}

This work presents one methods for airport conflict Hot Spots identification based on the Airport Surveillance Radar data. According to the identification results, which testified by rationality of the proposed method. It is of great significance to enhance safety operation of maneuver area and avoid aircraft or ground vehicle collision. However, the conflicts between aircrafts and vehicles, vehicles and vehicles should be taken into account of hot spot identification in the future.

\section{Reference}

[1] International Civil Aviation Organization. Procedures for Air Navigation Services-Air Traffic Management. Doc4444-ATM/501 [M]. Montreal: International Civil Aviation Organization. 2007:1-9.

[2] International Civil Aviation Organization. Manual on the Prevention of Runway Incursions (Doc9870 AN/463)[M]. Montreal: International Civil Aviation Organization(First Edition). 2006:3-20.

[3] Federal Aviation Administration. AIRPORT DIAGRAMD HOT SPOTS [M]. http://aeronav.faa.gov/afd/12Dec2013/NE_hotspot_12Dec2013.pdf, 2013.

[4] Huo Zhi-qin. Research on Hotspots Identification in Aerodrome through the Triple Selection Method [J]. China Safety Science Journal. 2012, 22(3): 92-96.

[5] Tang Wei-zhi. Study of data processing algorithm based on the surface movement radar. Nanjing: Nanjing University of Posts and Telecommunication Academic Degree Thesis. 2012.

[6] Dong Tian-gang, Li Xin-sheng. Mathematical models of ground motions simulation system for field surveillance radar. Computer Applications, 2008; 28(z2):378-379. 